\title{
Typing of Enterococcus spp. strains in 4 hospitals in the Małopolska region in Poland
}

\author{
Katarzyna Talaga 1, A-F, Dalma Odrowąż-Konduracka 2, B, F, Beata Paradowska 3, B, F, Barbara Jagiencarz-Starzec ${ }^{4, B, F}$, \\ Zdzisław Wolak ${ }^{4, B, F}$, Małgorzata Bulanda ${ }^{5, E, F}$, Anna Szczypta ${ }^{6, B, C, E, F}$ \\ 1 Department of Mycology, Chair of Microbiology, Faculty of Medicine, Jagiellonian University Medical College, Kraków, Poland \\ ${ }^{2}$ The Gabriel Narutowicz Municipal Specialistic Hospital, Kraków, Poland \\ ${ }^{3}$ The John Paul II Hospital, Kraków, Poland \\ ${ }^{4}$ St. Luke Provincial Hospital, Tarnów, Poland \\ ${ }^{5}$ Department of Epidemiology of Infections, Chair of Microbiology, Faculty of Medicine, Jagiellonian University Medical College, Kraków, Poland \\ ${ }^{6}$ Department of Health and Medical Sciences, Andrzej Frycz Modrzewski Krakow University, Poland \\ A - research concept and design; B - collection and/or assembly of data; $C$ - data analysis and interpretation; \\ $D$ - writing the article; $E$ - critical revision of the article; $F$ - final approval of the article
}

Address for correspondence

Katarzyna Talaga

E-mail: katarzyna.talaga@uj.edu.pl

Funding sources

This study was supported by a grant from

the Andrzej Frycz Modrzewski Krakow University,

Poland (No. WZiNM/DS/2/2015).

Conflict of interest

None declared

Received on July 29, 2016

Reviewed on September 29, 2016

Accepted on January 4, 2017

\section{Abstract}

Background. In the Małopolska province, the first case of vancomycin resistant enterococci (VRE) occurrence was an outbreak in 2001 caused by strains of the genus E. faecium carrying the vanA operon.

Objectives. The aim of this study is to determine the antimicrobial resistance and the occurrence of virulence determinants among Enterococcus spp. in patients hospitalized in the Małopolska region in 2015.

Material and methods. Antimicrobial susceptibility was determined by disc diffusion and the $E$ test. The presence of aminoglycoside and glycopeptide resistance genes and virulence genes (asal, gelE, cylA, esp, hyl) was investigated using multiplex polymerase chain reaction (PCR). Also, the presence of IS76 was investigated. The activity of gelatinase, cytolysin (hemolysin), and DNase was tested.

Results. All E. faecalis were susceptible to ampicillin, vancomycin, teicoplanin, linezolid and tigecycline. All E. faecium strains were susceptible to quinupristin-dalfopristin. Resistance to ampicillin and vancomycin was detected among all E. faecium isolates from hospitals C and D. 87.32\% of E. faecium presented high-level aminoglycoside-resistant (HLAR) phenotype, including 78.33\% of strains from hospital C and 100\% from hospital D. In hospital C (98.3\%) and D (96\%), resistance to ciprofloxacin, levofloxacin and norfloxacin was observed. Gene esp was detected in all E. faecium isolates and the majority of E. faecium isolates carried hyl (97\%). In E. faecalis, different combinations of virulence genes were detected. All analyzed E. faecium strains showed the presence of IS16 insertion element.

Conclusions. E. faecalis isolates were more susceptible to antimicrobials than E. faecium, which were largely multidrug-resistant. E. faecalis strains have diverse virulence factors. E. faecium showed a high percentage of hyl and esp genes and the presence of IS16.

Key words: virulence factors, healthcare-associated infections, Enterococcus, vancomycin resistant enterococci (VRE), antimicrobial susceptibility

DOI

$10.17219 /$ acem $/ 68265$

Copyright

Copyright by Author(s)

This is an article distributed under the terms of the

Creative Commons Attribution Non-Commercial License

(http://creativecommons.org/licenses/by-nc-nd/4.0/) 


\section{Introduction}

Enterococci are now recognized as a significant cause of healthcare-associated infections worldwide. ${ }^{1-3}$ The emergence of multidrug resistance (MDR), including high-level aminoglycoside-resistant (HLAR) enterococci and vancomycin-resistant enterococci (VRE), causes great difficulties in clinical antimicrobial therapy. ${ }^{4}$ VRE infections have been associated with higher mortality, longer hospital length of stay, and higher costs compared with vancomycin-susceptible isolates. 5,6 The first vancomycin-resistant $E$. faecium (VRE $f m$ ) strains carrying the $v a n A$ operon and $v a n B$ operon were reported in Poland in 1996 and 1999, respectively. ${ }^{7}$ In the Małopolska province, the first case of VRE occurrence was an outbreak caused by strains of the genus E. faecium carrying the $v a n A$ operon in 2001 . $^{8,9}$

The aim of this study is to determine the virulence factors and antibiotic resistance patterns of enterococcal isolates from 4 hospitals with different specialties from the Małopolska region.

\section{Material and methods}

\section{Hospital settings}

Pieces of information were obtained from hospitals from which enterococcal strains were isolated, regarding ward locations and body sites from which VRE and other enterococci were recovered. Material for the study was taken according to the following criteria: from patients with symptomatic infection as well as from patients admitted from other hospitals, Social Welfare Homes, Health Care Centers; patients who had previously been treated in another hospital and had been given broad-spectrum antibiotics; patients who had information about VRE colonization in their discharge card; the ones who were repeatedly hospitalized for 12 months.

\section{Characteristic of Enterococcus strains}

In this hospital-based study, a total of 154 Enterococcus strains isolated from 4 hospitals during the period from January 2015 through December 2015 were collected. Six of the samples were collected from hospital A, 13 samples were from B, 60 were from $C$, and 75 samples were acquired from hospital D. The enterococcal isolates were obtained from different specimens and were classified as colonization or infection. The study comprised consecutive, nonrepetitive enterococci isolates.

All strains had already been identified with the species level using conventional biochemical tests and with the VITEK-2 COMPACT fully automated microbiological system in hospital laboratories. The identification was confirmed by species-specific multiplex polymerase chain reaction (PCR) as described by Jackson (E. faecalis - 360 bp,
E. faecium - $215 \mathrm{bp}$ and E. avium - $368 \mathrm{bp}$ ) using the DNA samples of each Enterococcus strain isolated by Genomic Mini (A\&A Biotechnology, Gdynia, Poland). ${ }^{10}$

\section{Antibiotic susceptibility testing}

Susceptibility to antimicrobials was evaluated by the discdiffusion method (Oxoid, Basingstoke, England) according to manufacturer's procedure. The minimum inhibitory concentrations (MICs) of ampicillin, ciprofloxacin, tigecycline and linezolid were evaluated by the E tests method (bioMérieux, Marcy l'Etoile, France) according to the manufacturer's procedure.

The presence of HLAR phenotype, HLGR (high-level gentamicin resistance) and HLSR (high-level streptomycin resistance) phenotypes were identified by the disk susceptibility tests with streptomycin and gentamicin. VRE phenotype was detected by teicoplanin and vancomycin. The HLAR and VRE phenotypes were confirmed by the $\mathrm{E}$ tests method. All tests carried out using both methods were done on freshly prepared Mueller Hinton II Agar (Biocorp, Warszawa, Poland). The interpretation was performed in accordance with the recommendations of the European Committe on Antimicrobial Susceptibility Testing (EUCAST) guidelines. ${ }^{11}$

\section{Assay of gelatinase activity}

The production of gelatinase in Enterococcus strains was detected with the method described by Strzelecki. ${ }^{12}$ Gelatinase activity was observed as a transparent halo around the enterococcal colonies.

\section{Detection of antimicrobial resistance genes}

To detect genes responsible for HLAR phenotype in the genomes of all the strains resistant to gentamicin and/or streptomycin, multiplex PCR was applied according to the method of Vakulenko. ${ }^{13}$ The results of VRE testing by the phenotype method were confirmed by investigating vanA (732 bp) and vanB (635 bp) operons using the multiplex PCR as described by Biendo. ${ }^{14}$

Table 1. Characteristics of the studied hospitals

\begin{tabular}{|l|c|c|c|c|c|c|}
\hline \multirow{2}{*}{ Hospital } & \multicolumn{4}{|c|}{ Body sites } & \multicolumn{3}{c|}{ Units } \\
\cline { 2 - 7 } & blood & $\begin{array}{c}\text { rectal } \\
\text { swab }\end{array}$ & others* & ICU & surgery & others** \\
\hline A & 5 & 0 & 1 & 2 & 0 & 4 \\
\hline B & 0 & 0 & 13 & 2 & 7 & 4 \\
\hline C & 1 & 49 & 10 & 25 & 15 & 20 \\
\hline D & 2 & 55 & 18 & 57 & 7 & 11 \\
\hline Total & 8 & 104 & 42 & 86 & 29 & 39 \\
\hline
\end{tabular}

* pleural effusion, cerebrospinal fluid, urine, bronchial aspirates, surgical wound; ** Clinical Department of Interventional Cardiology, Department of Pulmonary Diseases, Department of Internal Medicine, Department of Orthopedic Trauma, Department of Neurology, Stroke Unit,

Department of Urology; ICU - intensive care unit. 


\section{Detection of virulence factor genes}

To detect the presence of genes encoding selected virulence factors (asa1, gelE, cylA, esp, hyl-375bp, $213 \mathrm{bp}$, $688 \mathrm{bp}, 510 \mathrm{bp}, 276 \mathrm{bp}$, respectively) in the enterococcal DNA, multiplex PCR was applied according to the methods of Vankerckhoven. ${ }^{15}$ The presence of quorum-sensing genes $\left(f s r A, f s r B, f_{s} r C\right)$ in the genomes of all the strains positive for the gelE gene was tested by multiplex PCR and was applied pursuant to Qin. ${ }^{16}$ The product sizes for $f_{s r} A$, $f_{s r} B$ and $f s r C$ were $484 \mathrm{bp}, 574 \mathrm{bp}$ and $580 \mathrm{bp}$, respectively.

\section{Assay of cytolysin (hemolysin) activity}

Cytolysin activity in Enterococcus strains was detected by the method described by Kowalska-Krochmal. ${ }^{17}$ The presence of a clear zone surrounding the studied strain colonies indicated the production of cytolysin.

\section{Assay of DNase activity}

DNase activity was detected on BD DNase Test Agar (Becton Dickinson, Oxford, England) described by Kowalska-Krochmal. ${ }^{17}$ DNase positive organisms will be surrounded by clear zones of depolymerized DNA. Colonies of DNase negative organisms will not show any clearing around the colonies.

\section{Detection of a specific mobile insertion element IS16 by PCR}

PCR was performed with primers encoding gene fragment of the insertion sequence IS16. ${ }^{18}$ The product sizes were $547 \mathrm{bp}$, which is specific for IS16 element.

\section{Results}

\section{Hospital settings}

Generally, over half of the strains were isolated from patients hospitalized in ICU (55.84\%), of which the highest proportion of enterococci was isolated from patients in hospital D and accounted for, respectively, the highest percentage $(76 \%)$ of strains in that hospital and among other hospitals.

In hospital A, no strain originated from a patient operated on, while in hospital B, it was the surgical unit that was the source of most strains (53.84\%). In general, most enterococci were isolated from perianal swabs, which resulted from a high rate of isolation in hospitals $C$ and $D$. In hospitals $\mathrm{A}$ and $\mathrm{B}$, no enterococci were isolated from rectal swabs. Only in hospital A, the biggest proportion of enterococci was isolated from the blood. At the remaining facilities, enterococci were significantly more often isolated from materials other that blood, as there is no procedure for screening for VRE on admission (Table 1).

\section{Distribution of Enterococcus species in various clinical specimens}

The highest prevalence of E. faecium was demonstrated in rectal swabs (75\%), followed by wounds (12.5\%), lower respiratory tract specimens $(5.8 \%)$, urine $(3.7 \%)$, blood (2.2\%), and others (0.8\%). The highest prevalence of E. faecalis was detected in wounds (47\%), followed by blood and lower respiratory tract specimens $(23.5 \%$ each), and cerebrospinal fluid (6\%). In our study, only one E. avium isolate was detected, and it was found in a postoperative wound swab.

Table 2. Prevalence of antimicrobial resistance in Enterococcus species isolated from hospitals A, B, C, and D (disk diffusion method)

\begin{tabular}{|c|c|c|c|c|c|c|c|c|}
\hline \multirow{3}{*}{ Antibiotic } & \multicolumn{8}{|c|}{ Hospital } \\
\hline & \multicolumn{2}{|c|}{ A } & \multicolumn{2}{|c|}{ B } & \multicolumn{2}{|c|}{ C } & \multicolumn{2}{|c|}{$\mathrm{D}$} \\
\hline & $\mathrm{S}$ & $\mathrm{R}$ & $S$ & $\mathrm{R}$ & $S$ & $\mathrm{R}$ & $S$ & $\mathrm{R}$ \\
\hline Ampicillin & $5(83.3)$ & $1(16.7)$ & $8(61.5)$ & $5(38.5)$ & $0(0)$ & $60(100)$ & $0(0)$ & $75(100)$ \\
\hline Ciprofloxacin & $4(66.7)$ & $2(33.3)$ & $6(46.2)$ & $7(53.8)$ & $1(1.7)$ & $59(98.3)$ & $3(4)$ & $72(96)$ \\
\hline Levofloxacin & $4(66.7)$ & $2(33.3)$ & $7(53.8)$ & $6(46.2)$ & $2(1.7)$ & $60(98.3)$ & $3(4)$ & $72(96)$ \\
\hline Norfloxacin & $4(66.7)$ & $2(33.3)$ & $6(46.2)$ & $7(53.8)$ & $3(1.7)$ & $61(98.3)$ & $3(4)$ & $72(96)$ \\
\hline Gentamicin & $3(50)$ & $3(50)$ & $6(46.2)$ & $7(53.8)$ & $3(5)$ & $57(95)$ & $0(0)$ & 75 (100) \\
\hline Streptomycin & $6(100)$ & $0(0)$ & $8(61.5)$ & $5(38.5)$ & $8(13.3)$ & $52(86.7)$ & $9(12)$ & $66(88)$ \\
\hline Vancomycin & $6(100)$ & $0(0)$ & $12(92.3)$ & $1(7.7)$ & $0(0)$ & $60(100)$ & $0(0)$ & $75(100)$ \\
\hline Teicoplanin & $6(100)$ & $0(0)$ & $12(92.3)$ & $2(7.7)$ & $15(25)$ & $45(75)$ & 70 (93.3) & $5(6.7)$ \\
\hline Tigecycline & $6(100)$ & $0(0)$ & $13(100)$ & $0(0)$ & $60(100)$ & $0(0)$ & 75 (100) & $0(0)$ \\
\hline Linezolid & $6(100)$ & $0(0)$ & $13(100)$ & $0(0)$ & $60(100)$ & $0(0)$ & 75 (100) & $0(0)$ \\
\hline Quinupristin-dalfopristin & $1^{*}(100)$ & $0(0)$ & $3^{*}(100)$ & $0(0)$ & $60 *(100)$ & $0(0)$ & $75^{*}(100)$ & $0(0)$ \\
\hline
\end{tabular}

$\mathrm{S}$ - susceptible; $\mathrm{R}$ - resistant; $\mathrm{n}(\%)$ - number (percentage) of strains; * quinupristin-dalfopristin was evaluated for $E$. faecium only. 


\section{Comparison of antimicrobial resistance between $E$. faecium and $E$. faecalis and antimicrobial resistance in Enterococcus species isolated from various hospitals}

The E test method confirmed the resistance to antimicrobials shown by the disc diffusion method (vancomycin, teicoplanin, ampicillin, gentamicin, ciprofloxacin, linezolid, and tigecycline) (Tables 2, 3). A strain resistant to linezolid and tigecycline was not reported among the tested enterococcal strains. All E. faecalis were also susceptible to ampicillin, vancomycin and teicoplanin. HLAR, HLGR and HLSR phenotypes were present in $33.3 \%, 20 \%$ and $6.6 \%$ of $E$. faecalis strains, respectively. All enterococci from $E$. faecium species were also susceptible to quinupristin-dalfopristin. Among all E. faecium isolates from hospitals $C$ and $D$, resistance to ampicillin and vancomycin was detected. A high percentage of E. faecium (87.32\%) presented HLAR phenotype, including 78.33\% of strains from hospital C and 100\% from D. Furthermore, in hospital D, HLSR (1.67\%) and HLGR (10\%) were detected in E. faecium. Also, very high rates of resistance to ciprofloxacin and 2 other fluoroquinolones (levofloxacin and norfloxacin) were observed in hospitals C (98.3\%) and D (96\%). In our study, E. faecalis isolates were more susceptible to antimicrobials than E. faecium, which were largely multidrug-resistant. Detailed results are shown in Tables 2 and 3. In hospitals A and B, MDR was not reported among the tested Enterococcus strains. In hospitals C and D, 95\% and 96\%, respectively, were classified as MDR strains.

\section{Prevalence of resistance genes}

In the study group there were 136 gentamicin resistance strains containing the $\operatorname{aac}\left(6^{\prime}\right)-\operatorname{Ie}$-aph(2")-Ia gene, which encodes the bifunctional enzyme AAC(6')-APH(2"), and 6 strains encoding the $a p h(2$ ")-Id gene that also mediates resistance to gentamicin. All 123 streptomycin resistance strains contained the aph(3')-IIIa gene. 115 vanB genes and only 21 vanA genes were detected in enterococci. PCR analysis confirmed the phenotypic analysis.

\section{Detection of virulence factor genes}

Esp genes were detected in all E. faecium isolates and the majority of E. faecium isolates carried hyl (97\%), in contrast to $E$. faecalis strains, in which different combinations of asal, esp, gelE, and $c y l A$ genes were detected (Fig. 1).

\section{Phenotypic analysis of virulence factors}

In hospitals $\mathrm{A}$ and $\mathrm{B}$, where $E$. faecalis constituted the majority of the isolated strains, cytolysin as well as gelatinase were produced. $100 \%$ of the gene responsible for

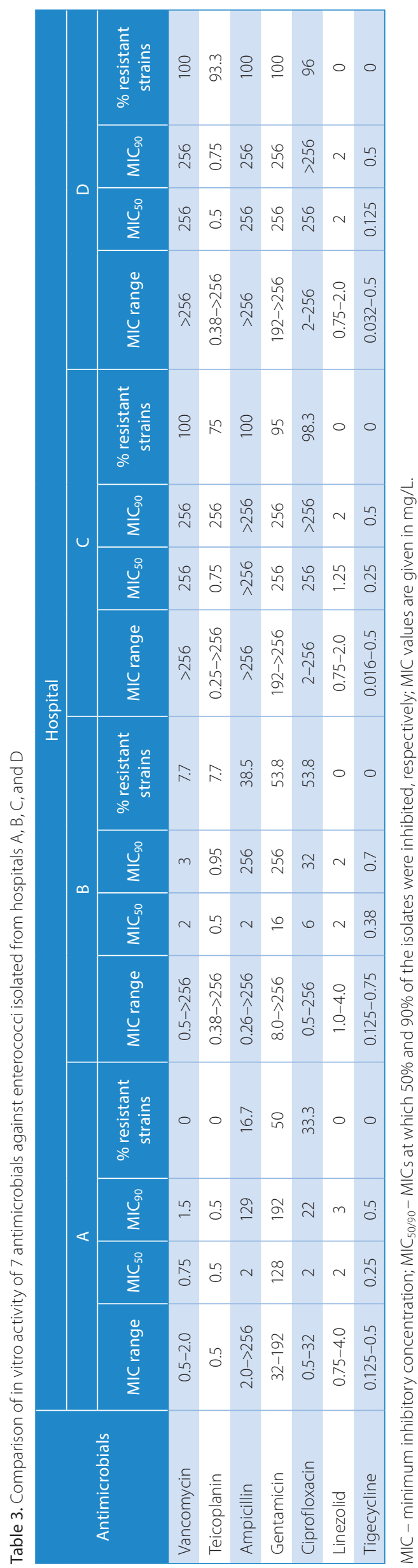


producing cytolysin underwent expression. The activity of gelatinase was detected only for 5 strains that had all 3 regulator genes $(f s r A, f s r B$ and $f s r C)$ from 9 enterococci containing the gene encoding gelatinase. No DNase activity was observed in any of the tested strains from all the hospitals (Table 4).

\section{Detection of a specific mobile insertion element IS16}

Molecular analysis of all analyzed E. faecium strains showed the presence of a gene fragment specific for IS16 insertion element.

\section{Discussion}

In our study, the majority of patients were hospitalized in ICU, which is similar to other reports and seems to be an important risk factor for enterococci infections. ${ }^{19,20}$

Table 4. Distribution of cytolysin (hemolysin), gelatinase and DNase activity in the genus Enterococcus isolates from hospitals A, B, C, and D

\begin{tabular}{|l|c|c|c|}
\multirow{2}{*}{ Hospital } & \multicolumn{3}{|c|}{ Virulence determinants } \\
\cline { 2 - 4 } & cytolysin & gelatinase & DNase \\
\hline A & 1 & 2 & 0 \\
\hline B & 2 & 3 & 0 \\
\hline C & 0 & 0 & 0 \\
\hline D & 0 & 0 & 0 \\
\hline Total & 3 & 5 & 0 \\
\hline
\end{tabular}

\section{Antimicrobial resistance}

Our study confirms that E. faecalis is still a rare reservoir of acquired vancomycin resistance, and this trend is well-known in our country and in Europe. ${ }^{2,21}$ The majority of E. faecalis strains were susceptible to ampicillin and other antimicrobials, and it seems that E. faecalis species is not a therapeutic problem in hospitals in Małopolska.

The major determinant of vancomycin resistance among Polish VREfm is still the VanA gene cluster, which is also most prevalent in some European counties. ${ }^{2,19,22}$ However, in our study, E. faecium VanA phenotype strains were in the minority, while VanB phenotype isolates were in the majority. This seems to be the characteristic feature for VRE $f m$ from the Małopolska region, because in other provinces VanA phenotype is predominant. $8,23,24$ In Małopolska, in recent years, the presence of both VanA and VanB phenotypes has been described. ${ }^{25-27}$ All VREfm strains isolated in this study were MDR and this is a problem for the therapeutic management of patients. The most important problem with the multidrug resistance of the studied strains is that they are able to easily acquire (by plasmids and/or transposon transfer) new resistance traits between enterococci.

In our research, we isolated only 1 strain which belonged to the E. avium species and included the HLAR phenotype but was susceptible to other antimicrobials. Therefore, it seems that other enterococcal species are not a threat to the therapeutic situation in the Małopolska region nowadays. In our study, only a few E. faecalis strains and a large group of E. faecium strains, including all VRE $f m$ isolates, were resistant to ampicillin. It is a very disturbing and

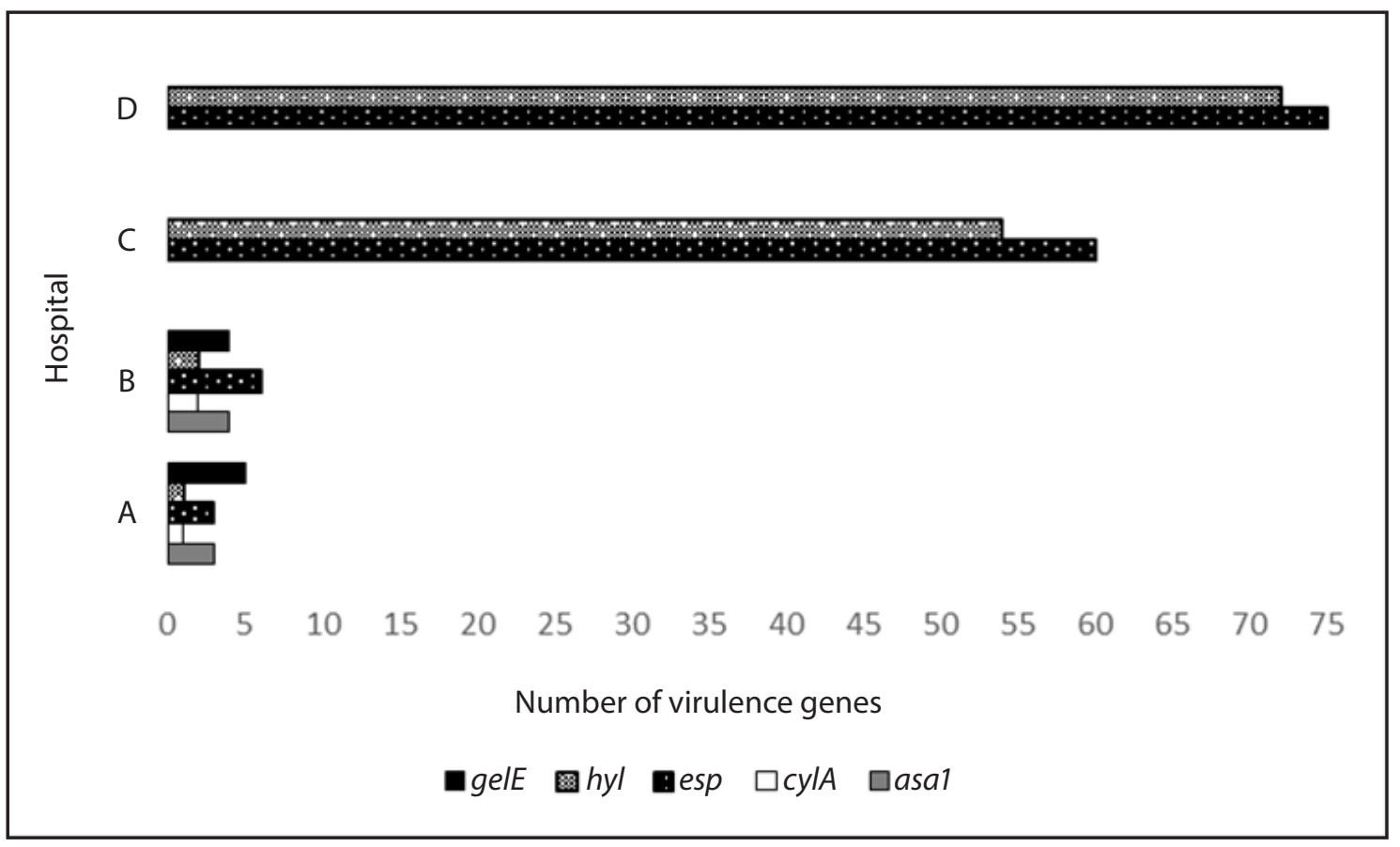

Fig. 1. Occurrence of virulence determinants asal, gelE, cylA, esp and hyl in enterococci isolates from hospitals $A, B, C$, and D 
dangerous situation, because $\beta$-lactam antibiotics are a vital group of drugs employed for the treatment of infections caused by enterococci. Ampicillin resistance also indicates resistance to amoxicillin, piperacillin and preparations combined with $\beta$-lactamase (ampicillin/sulbactam, amoxicillin/clavulanic acid, piperacillin/tazobactam).${ }^{28}$ Moreover, it is well known that ampicillin-resistant E. faecium are a precursor of multidrug-resistant strains, including VRE, and are widespread in Polish and European hospitals. ${ }^{2,19}$ Enterococci are inherently resistant to low concentrations of aminoglycosides, which is associated with reduced permeability of these antibiotics through the cell wall. ${ }^{29}$ In agreement with other studies from Poland, also ours, the spread of the $\operatorname{aac}\left(6^{\prime}\right)-I e-a p h\left(2^{\prime \prime}\right)-I a$ gene, which encodes the bifunctional enzyme $\mathrm{AAC}\left(6^{\prime}\right)-\mathrm{APH}\left(2^{\prime \prime}\right)$ and eliminates the synergistic effect between $\beta$-lactams or glycopeptide antibiotics and aminoglycosides, was responsible for high-level resistance to gentamicin among the majority of enterococci. ${ }^{22,28}$ Thus, it precludes its therapeutic application in severe infections caused by enterococci, including bacteremia and endocarditis. ${ }^{28,29}$ If the strains are resistant to high concentrations of gentamicin with simultaneous sensitivity to streptomycin (HLGR), streptomycin can be used in combination with $\beta$-lactams and glycopeptides. If the strains display HLSR phenotype, which means that they are resistant to high concentrations of streptomycin with simultaneous sensitivity to gentamicin, streptomycin therapy can be applied combined with $\beta$-lactams and glycopeptides, since the therapeutic effect is present. Detection of resistance to high concentrations of gentamicin and streptomycin at the same time (HLAR) means that there is no synergy of aminoglycosides with $\beta$-lactams and glycopeptides, which was found in a high degree in the strains examined by us. ${ }^{28,29}$ For years, a tendency that has appeared among enterococci, also in Poland, regards their growing resistance to fluoroquinolones. In hospital A, this regularity is poorly marked (only $33.3 \%$ of resistant strains). In hospital B, it increases to approx. $50 \%$ of resistant strains, but it is best marked by a high resistance to ciprofloxacin, levofloxacin and norfloxacin in the enterococci investigated in hospitals $C$ and $D$. This also proves that a very high percentage of resistant strains is common among the species E. faecium, which is also described in other studies originating in Poland. ${ }^{19,28}$ Quinupristin-dalfopristin (Q/D) (with the exception of the $E$. faecalis species), linezolid and tigecycline have bacteriostatic activity against VRE, so they are recommended for the treatment of various infections caused by strains simultaneously resistant to several groups of antibiotics, such as ampicillin, glycopeptides, and a high concentration of aminoglycosides. Our data suggests that these antimicrobials may be effective therapeutic options for the treatment of serious infections caused by the studied enterococci strains. Unfortunately, Q/D treatment has failures and adverse effects, whereas acquired resistance to linezolid has been observed in enterococci, but this phenomenon is still rare and associated with the duration of previous linezolid therapy.

\section{Virulence factors}

Enterococci displaying virulence factors are more likely to cause an infection than the strains devoid of them. There are factors that facilitate colonization and those that facilitate the infection of previously colonized tissue. The majority of VREfm strains from hospitals $\mathrm{C}$ and $\mathrm{D}$ both contained $e s p$ and $h y l_{E f m}$ genes. Hyaluronidase (encoded by the $h y l$ gene) contributes to the destruction of connective tissue and thus makes it easier for bacteria to spread in an infected organism. ${ }^{15}$ The enterococcal surface protein Esp (encoded by esp) is associated with increased virulence, colonization and persistence in the urinary tract and biofilm formation. ${ }^{15,18}$ In hospitals $A$ and $B$, the situation was more diverse, and gelE, asa1, esp, $h y l_{E f m}$, and $c y l A$ genes in different combinations were detected in the enterococcal isolates. Gelatinase (encoded by gelE) has the ability to hydrolyze collagen, gelatin and small peptides. ${ }^{15,18}$ Aggregation substance (encoded by asa1) facilitates the adhesion of enterococci to host cells, supports aggregation and facilitates the survival of bacteria in macrophages. Cytolysin has bacteriocin activity and the ability to lyse certain eukaryotic erythrocytes and gram-positive bacterial cells. ${ }^{15,18}$ Among the studied enterococci, there were also strains which did not have virulence determinants. Moreover, like other researchers, we did not find DNase activity in either E. faecalis, E. faecium or E. avium isolates. ${ }^{17}$ Therefore, it seems that this is not a virulence factor that occurs in enterococci.

\section{The correlation between drug resistance and virulence genes}

Nowadays, in European hospitals, the vancomycin-susceptible enterocci are replaced by high-level ampicillin and ciprofloxacin resistance, HLAR and VRE phenotype simultaneously, which are typical of hospital-acquired VREfm. The E. faecium strains examined in our study also shared other subpopulation characteristics of hospital-acquired strains, such as the presence of esp, hyl and IS16. ${ }^{18,30}$ Phenotypic and molecular characterization of the E. faecium isolates tested coming from hospitals $\mathrm{C}$ and $\mathrm{D}$ corresponds to the characteristics of strains with high epidemic potential occurring in hospitals in Europe (Clonal Complex CC17). The specific mobile insertion element IS16 is highly specific for predicting hospital-associated strain types. ${ }^{30}$ In our study, all strains belonging to the E. faecium species showed the presence of IS16, which is a similar result to other studies from Poland. ${ }^{19,22,23,30}$

\section{Conclusions}

The E. faecalis strains that appear in Małopolska hospitals are largely sensitive to antibiotics and have a variable amount of virulence factors. The E. faecalis species were 
isolated less frequently than E. faecium from patients hospitalized in Małopolska hospitals. In contrast, the strains of the species E. faecium are a more uniform group with resistance to a number of significant therapeutic drug types, such as ampicillin, high concentrations of aminoglycosides, fluoroquinolones and glycopeptides (also more often displaying the VanB phenotype). VREfm strains also have a high proportion of $h y l$ and esp genes, which is characteristic of hospital strains of enterococci.

\section{Reference}

1. Panesso D, Reyes J, Rincón S, et al. Molecular epidemiology of vancomycin-resistant Enterococcus faecium: A prospective, multicenter study in South American hospitals. J Clin Microbiol. 2010;48:1562-1569.

2. Werner G, Coque TM, Hammerum AM, et al. Emergence and spread of vancomycin resistance among enterococci in Europe. Euro Surveill. 2008;13(47): pii = 19046. Available online: http://www.eurosurveillance.org/ViewArticle.aspx?Articleld = 19046. Accessed July 20, 2016

3. Patel R, Gallagher JC. Vancomycin-resistant enterococcal bacteremia pharmacotherapy. Ann Pharmacother. 2015;49:69-85.

4. Iosifidis E, Evdoridou I, Agakidou E, et al. Vancomycin-resistant Enterococcus outbreak in a neonatal intensive care unit: Epidemiology, molecular analysis and risk factors. Am JInfect Control. 2013;41:857-861.

5. Jia W, Li G, Wang W. Prevalence and antimicrobial resistance of Enterococcus species: A hospital-based study in China. Int J Environ Res Public Health. 2014;11:3424-3442.

6. O'Driscoll T, Crank CW. Vancomycin-resistant enterococcal infections: Epidemiology, clinical manifestations, and optimal management. Infect and Drug Resist. 2015;8:217-230.

7. Hryniewicz W, Szczypa K, Bronk M, Samet A, Hellmann A, Trzcinski K. First report of vancomycin-resistant Enterococcus faecium isolated in Poland. Clin Microbiol Infect. 1999;5:503-505.

8. Kawalec M, Gniadkowski M, Zielinska U, Kłos W, Hryniewicz W. Vancomycin-resistant Enterococcus faecium strain carrying the vanB2 gene variant in a Polish hospital. J Clin Microbiol. 2001;39:811-815.

9. Kędzierska J, Węgrzyn J, Skotnicki AB, Kędzierska A. Therapeutic and epidemiological aspects of infections with vancomycin-resistant strains from the genus Enterococcus in patients with haematological neoplastic disorders. Acta Haematologica Polonica. 2003;34:187-199.

10. Jackson CR, Fedorka-Cray PJ, Barrett JB. Use of a genus- and speciesspecific multiplex PCR for identification of enterococci. J Clin Microbiol. 2004;42:3558-3565.

11. The European Committee on Antimicrobial Susceptibility Testing. Breakpoints tables for interpretation of MICs and zones diameters. Version 5.0, 2015. Available online: http://www.eucast.org. Accessed July 21, 2016.

12. Strzelecki J, Hryniewicz W, Sadowy E. Gelatinase-associated phenotypes and genotypesamong clinical isolates of Enterococcus faecalis in Poland. Pol J Microbiol. 2011;60:287-292.

13. Vakulenko SB, Donabedian SM, Voskresenskiy AM, Zervos MJ, Lerner SA, Chow JW. Multiplex PCR for detection of aminoglycoside resistance genes in enterococci. Antimicrob Agents Chemother. 2003;47:1423-1426.

14. Biendo M, Adjidé C, Castelain S, et al. Molecular characterization of glycopeptide-resistant enterococci from hospitals of the Picardy Region (France). Int J Microbiol. 2010;2010:150464.

15. Vankerckhoven V, Van Autgaerden T, Vael C, et al. Development of a multiplex PCR for the detection of asal, gelE, cylA, esp, and hyl genes in enterococci and survey for virulence determinants among European hospital isolates of Enterococcus faecium. J Clin Microbiol. 2004;42:4473-4479.

16. Qin X, Singh KV, Weinstock GM, Murray BE. Effects of Enterococcus faecalis fsr genes on production of gelatinase and a serine protease and virulence. Infect Immun. 2000;68:2579-2586.

17. Kowalska-Krochmal B, Dworniczek E, Dolna I, et al. Resistance patterns and occurrence of virulence determinants among GRE strains in southwestern Poland. Adv Med Sci. 2011;56:304-310.

18. Werner G, Fleige C, Geringer U, Schaik W, Klare I, Witte W. IS element IS16 as a molecular screening tool to identify hospital-associated strains of Enterococcus faecium. BMC Infect Dis. 2011;31:11-80.
19. Gawryszewska I, Żabicka D, Bojarska K, Malinowska K, Hryniewicz W, Sadowy E. Invasive enterococcal infections in Poland: The current epidemiological situation. Eur J Clin Microbiol Infect Dis. 2016;35:847-856.

20. Pinhold $\mathrm{M}$, Larner-Svensson $\mathrm{H}$, Littauer $\mathrm{P}$, et al. Multiple hospital outbreaks of vanA Enterococcus faecium in Denmark, 2012-13, investigated by WGS, MLST and PFGE. J Antimicrob Chemother. 2015;70:2474-2482.

21. Kuch A, Willems RJ, Werner G, et al. Insight into antimicrobial susceptibility and population structure of contemporary human Enterococcus faecalis isolates from Europe. J Antimicrob Chemother. 2012;67:551-558.

22. Sadowy E, Sieńko A, Gawryszewska I, Bojarska A, Malinowska K, Hryniewicz W. High abundance and diversity of antimicrobial resistance determinants among early vancomycinresistant Enterococcus faecium in Poland. Eur J Clin Microbiol Infect Dis. 2013;32:1193-11203.

23. Wardal E, Markowska K, Zabicka D, et al. Molecular analysis of vanA outbreak of Enterococcus faecium in two Warsaw hospitals: The importance of mobile genetic elements. Biomed Res Int. 2014;2014:575367.

24. Młynarczyk G, Grzybowska W, Młynarczyk A, et al. Significant increase in the isolation of glycopeptide-resistant enterococci from patients hospitalized in the transplant surgery ward in 2004-2005. Transplant Proc. 2007;39:2883-2885.

25. Kawalec M, Kedzierska J, Gajda A, et al. Hospital outbreak of vancomycin-resistant enterococci caused by a single clone of Enterococcus raffinosus and several clones of Enterococcus faecium. Clin Microbiol Infect. 2007;13:893-901.

26. Kedzierska J, Wegrzyn J, Skotnicki AB, Skop A, Pawliszyn W. Infections with VanB phenotype Enterococcus faecium and E. faecalis in patients with immune deficiency during the course of hematologic neoplasms. Med Dosw Mikrobiol. 2003;55:11-24.

27. Kawalec M, Gniadkowski M, Kedzierska J, Skotnicki A, Fiett J, Hryniewicz W. Selection of a teicoplanin-resistant Enterococcus faecium mutant during an outbreak caused by vancomycin-resistant enterococci with the VanB phenotype. J Clin Microbiol. 2001;39:4274-4282.

28. Lisiecki P. Antibiotic resistance and siderophore production in enterococci. Med Dosw Mikrobiol. 2014;66:1-10.

29. Hollenbeck BL, Rice LB. Intrinsic and acquired resistance mechanisms in enterococcus. Virulence. 2012;3:421-433.

30. Chabros L, Szymanek-Majchrzak K, Młynarczyk A, et al. Evaluation of the prevalence of insertion element IS16 in vancomycin-resistant enterococci strains of Enterococcus faecium isolated from transplantology patients from a Warsaw hospital between 2010 and 2012 . Transplant Proc. 2014;46:2583-2585. 